\title{
Neurofibromatosis y tumor estromal gastrointestinal diagnosticado por enteroscopia anterógrada. Reporte de caso
}

\author{
Félix Téllez-Ávila ${ }^{*}$ y Fredy Chable-Montero ${ }^{2}$
}

${ }^{1}$ Departamento de Endoscopia Gastrointestinal, Instituto Nacional de Ciencias Médicas y Nutrición Salvador Zubirán; ²Unidad de Patología, HoSpital San Ángel Inn Universidad. Ciudad de México, México

\section{Resumen}

La enfermedad de von Recklinhausen, o neurofibromatosis tipo 1, es una enfermedad autosómica dominante poco frecüente que puede presentar diferentes manifestaciones gastrointestinales, que van desde cuadros obstructivos a sangrado intestinal. Se presenta el caso de un paciente con cuadros recurrentes de sangrado gastrointestinal, pérdida de peso y hepatopatía crónica en quien se diagnosticó un tumor del estroma gastrointestinal.

Palabras clave: Neurofibromatosis. Tumor estromal. Enteroscopia. México.

GIST in a patient with neurofibromatosis type 1 diagnosed by anterograde enteroscopy. Case report
Abstract
Von Recklinghausens's disease, or neurofibromatosis type 1, is a rare autosomal dominant disease which may presentif- ferent gastrointestinal manifestations ranging from obstructive symptomatology to gastrointestinal bleeding. We reporethe case of a patient with recurrent episodes of gastrointestinal bleeding, weight loss and chronic liver disease in whom a gastrointestinal stromal tumor was diagnosed by enteroscopy.
Key words: Neurofibromatosis. Estromal tumor. Enteroscopy. Mexico.

\section{Introducción}
La neurofibromatosis tipo 1 (NF1), conocida como enfermedad de von Recklinghausen, es una anomalía que se presenta en el cromosoma 17q11.21. Tiene una incidencia de 1:3,000 nacimientos y una prevalencia de $1: 5,000^{3}$. Se caracteriza por ser una enfermedad progre- siva, autosómica dominante, multisistémica, que ade- más posee una penetrancia completa y expresividad
variable ${ }^{1,4}$. En esta enfermedad el defecto se encuentra en el gen que codifica la proteína neurofibromina, Una proteína que controla la proliferación celular al inactivar el p21 RAS y la vía de la MAP cinasa ${ }^{2,4}$. Existen muchas mutaciones de novo en este gen, es por eso que hásta un $50 \%$ de los pacientes no tienen historia familiar de la enfermedad².
Los tumores estromales de sistema digestivo (GIST) son neoplasias mesenquimales submucosas que se
Correspondencia:
*Félix Téllez Ávila
E-mail: felixtelleza@gmail.com
DOl: 10.24875/END.M19000170
Fecha de recepción: 04-07-2019
Fecha de aceptación: 03-09-2019

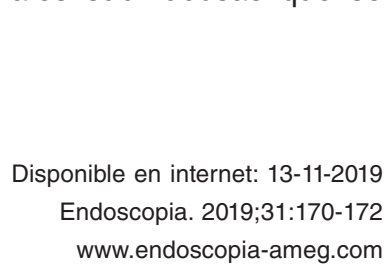


originan en la muscular propia a partir de las células de Cajal ${ }^{1,4,5}$. Se caracterizan por la expresión inmunohistoquímica del receptor transmembrana del protooncogén c-kit, así como de la expresión del marcador CD34 $4^{1,5}$. Representan hasta el $1.3 \%$ de todos los tumores del tracto gastrointestinal, el $1-3 \%$ de las neoplasias gástricas y el $20 \%$ de las del intestino delgado ${ }^{1}$.

\section{Presentación de caso}

Mujer de 71 años, originaria de Mexicali y residente en el distrito federal. Entre sus antecedentes patológicos refiere una amigdalectomía, colecistectomía laparoscópica e histerectomía. Fue diagnosticada con neurofibromatosis en su juventud. Además refiere hipertensión arterial e infección por virus de hepatitis C.

Acude con un cuadro de varios años de evolución caracterizados por evacuaciones melénicas. Estas se presentan 2 a 3 veces por año y tienen una duración de hasta 2 o 3 semanas. Desde hace 3 meses presenta astenia, adinamia, náuseas y vómitos. En agosto del 2014 presenta un nuevo episodio de melena. Estas se presentan 2 por día, durante 2 a 3 días y se asocia con una pérdida de peso de $5 \mathrm{~kg}$. Se realizó una biometría hemática y se documenta una hemoglobina de $7.9 \mathrm{~g} / \mathrm{dl}$, hematocrito de $22.9 \%$ y plaquetas 203.000 cél. $/ \mathrm{mm}^{3}$. No se apreciaban alteraciones en valores de química sanguínea o pruebas de funcionamiento hepático. Se solicita un estudio de imagen (Fig. 1), donde se reporta un aumento anormal del metabolismo glucolítico en yeyuno e íleon terminal. Con los hallazgos, se realizó enteroscopia anterógrada y retrógrada de un solo balón en donde se observaron erosiones lineales menores de $5 \mathrm{~mm}$ en fondo y cuerpo de estómago. A $70 \mathrm{~cm}$, después del ángulo de Treitz, se observó una lesión de aproximadamente $1.5 \mathrm{~cm}$, firme. Se tomó biopsia utilizando técnica de biopsia sobre biopsia (Fig. 2). En la enteroscopia retrógrada se observó un aumento en el volumen de la válvula ileocecal a expensas de su tejido blando. La evaluación de patología mostró una enteritis crónica leve con hemorragia focal de la lámina propia, sin atrofia de vellosidades intestinales; en la lesión tumoral se realizó inmunohistoquímica, que reporta CD34 positivo, CD117 positivo, DOG.1 positivo y desmina negativa, compatible con tumor del estroma gastrointestinal fusocelular (Fig. 3). La paciente egresó del hospital para manejo ambulatorio y abordaje de su hepatopatía crónica.

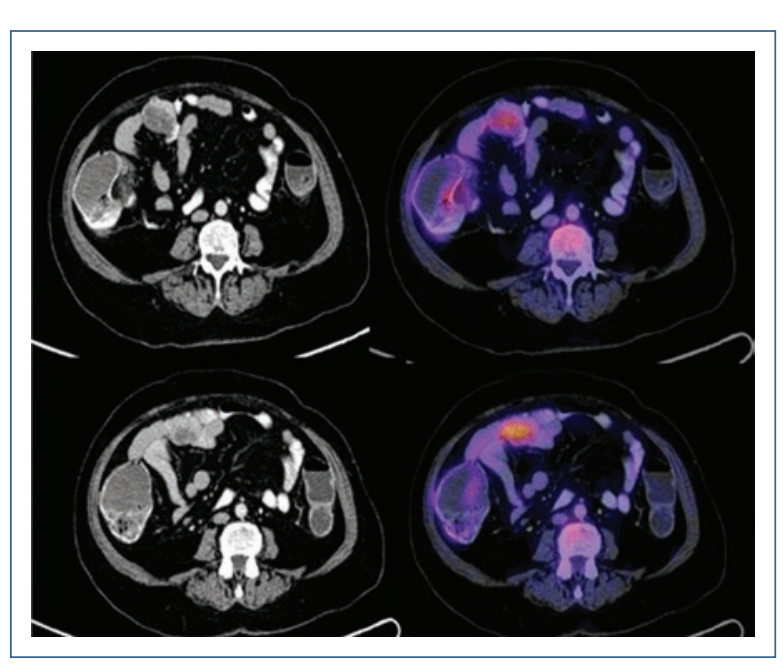

Figura 1. Aumento anormal del metabolismo glucolíticen en yeyuno e íleon terminal.

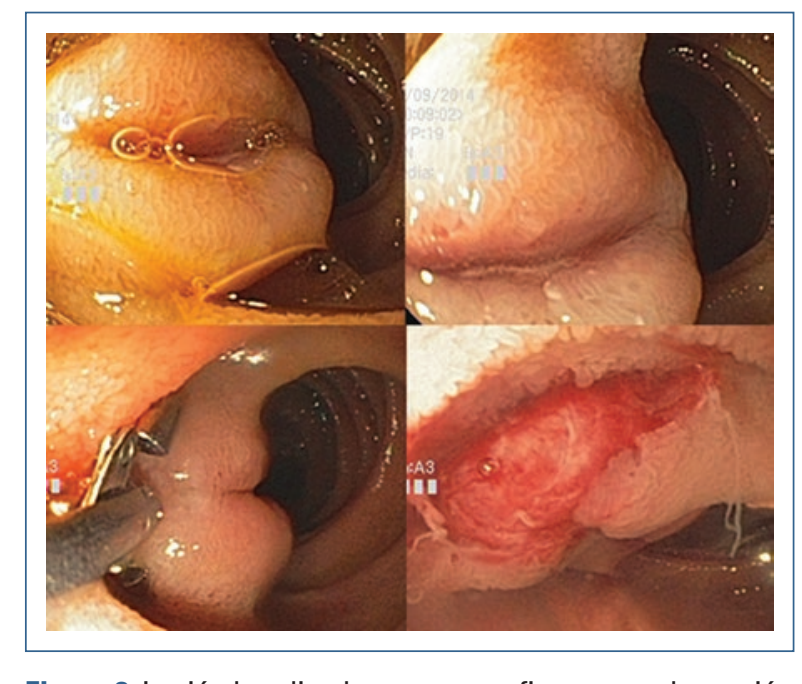

Figura 2. Lesión localizada en yeyuno, firme, con ulceración superficial. Se tomó biopsia utilizando técnica de biopssia sobre biopsia.

\section{Discusión}

Los pacientes con NF1 pueden presentar difereñtes manifestaciones gastrointestinales. La frecuencia de estas puede ser del 5 al 25\%2,4. Existen difereñtes formas de implicación digestiva: a) hiperplasia de la submucosa y de las células de Schwann de los plexos nerviosos mioentéricos; b) formación de diferentes variedades de tumores estromales; c) mayor prevaleñcia de carcinoides en la zona periampular, y d) mayor predisposición que la población general a padecer adenocarcinomas ${ }^{1,6}$. 


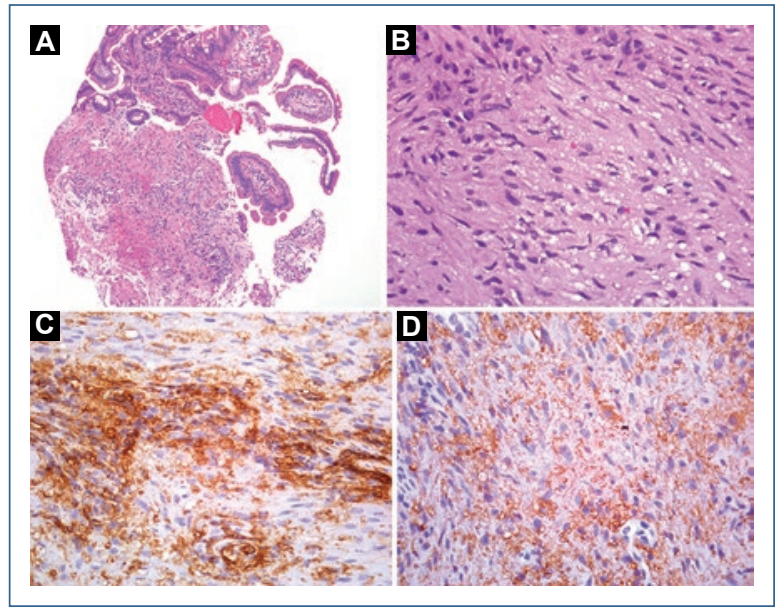

Figura 3. A: la mucosa yeyunal mostró una neoplasia mesenquimal subepitelial benigna. B: el tumor estaba compuesto de células fusiformes con atipia leve y citoplasma vacuolado. C: las células neoplásicas fueron CD34 positivo. D: las células neoplásicas fueron CD117 positivo. Además, fue positivo para DOG.1 (no mostrado).

Los GIST en este tipo de pacientes se pueden presentar de forma tardía en la vida, incluso mucho después de los síntomas cutáneos ${ }^{2}$. Presentan una ligera predominancia en mujeres ${ }^{6}$. Existen diferentes expresiones clínicas de estas lesiones. Se considera que una tercera parte de las lesiones menores de $3 \mathrm{~cm}$ cursan con sintomatología ${ }^{1}$, es así que hasta un 50\% de los pacientes pueden permanecer asintomáticos, mientras que si son lesiones sintomáticas se pueden presentar con hemorragia del tubo digestivo secundaria a ulceración del tumor y síndrome anémico, así como dispepsia, náuseas, vómitos, dolor abdominal, masa palpable, obstrucción intestinal con intususcepción u obstrucción renoureteral ${ }^{6,7}$.

Debido a su localización (hasta un 6\% son duodenales y un $4 \%$ son yeyunoileales) ${ }^{2}$, la enteroscopia es una herramienta diagnóstica que permite acceder a áreas del intestino a las que previamente no era posible. Cuando se aprecian estas lesiones, como en nuestra paciente, se pueden observar áreas con erosiones, zonas de hemorragia, degeneración quística o necrosis ${ }^{1,3}$.
Si bien son consideradas lesiones submucosas, si se obtienen muestras mediante una técnica de biopsia sobre biopsia es posible realizar el diagnóstico. En los hallazgos histológicos, estas lesiones muestran céiulas fusiformes con eosinófilos PAS-positivos. Un detalleimportante en estos tumores es su falta de mutaciones en el gen KIT (presente en el $80-85 \%$ de los GIST) y de receptor del factor de crecimiento derivado de plaquetas alfa (PDGFR- $\alpha)^{4,8}$. Cuando se aprecia la âsociación de las dos enfermedades la mayoría presénta un fenotipo salvaje. La importancia de este hallazgo radica en su sensibilidad al imatinib.

Los pacientes con NF1 pueden tener diferentes manifestaciones gastrointestinales, una de ellas pứede ser un GIST. Es importante tenerlas presentes a fin de que se pueda realizar un diagnóstico oportuno.

\section{Financiamiento}

La presente investigación no ha recibido ayudašespecíficas provenientes de agencias del sector púbtico, sector comercial o entidades sin ánimo de lucro.

\section{Conflicto de intereses}

Los autores declaran no tener ningún conflicto de intereses.

\section{Bibliografía}

1. Merino Rodríguez B, Vega Catalina Rodríguez M, Bachiller L, Clemente Ricote G. [Upper gastrointestinal bleeding secondary to a jejunal stromal tumor in a patient with neurofibromatosis type 1]. Gastroenterol Hepatol. 2009;32:287-90.

2. Agaimy A, Vassos N, Croner RS. Gastrointestinal manifestations of neurofibromatosis type 1 (Recklinghausen's disease): clinicopathological spectrum with pathogenetic considerations. 2012;5:852-62.

3. Guillaud O, Dumortier J, Bringuier P, Saurin JPG. Tumeurs stromales gastro-intestinales (GIST) multiples chez un malade atteint d'une maladie de Recklinghausen. Gastroenterol Clin Biol. 2006;30:320-4.

4. Sawalhi S, Al-Harbi K, Raghib Z, Abdelrahman Al, Al-Hujaily A. Behavior of advanced gastrointestinal stromal tumor in a patient with von-Recklinghausen disease: Case report. World J Clin Oncol. 2013;4:70-4. ㅇ

5. Shinomura Y, Kinoshita K, Tsutsui S, Hirota S. Pathophysiology, diagnosis, and treatment of gastrointestinal stromal tumors. J Gastroenterol. $2005 ; 40: 775-80$

6. Saha SB, Parmar R, Mandal A. Small bowel obstruction in a neurofibromatosis patient-A rare presentation of gastro-intestinal stromal tumors (GISTs): Case report and literature review. Indian J Surg. 2013;75:415-7.

7. Joensuu $\mathrm{H}$, Hohenberger P, Corless CL. Gastrointestinal stromal tumour. Lancet. 2013;382:973-83.

8. O'Regan KN, Shinagare AB, Saboo SS, Ramaiya NH, Jagannathan JP, Tirumani SH. Gastrointestinal stromal tumors (GIST): lesser knownfacts. Clin Imaging. 2013;37:821-9. 